\title{
Research Challenges in Complex Large Scale Networks and Cyber Physical Systems
}

\author{
Ty Znati \\ National Science Foundation, USA \\ tznati@nsf.gov
}

\begin{abstract}
Unprecedented advances in technology are revolutionizing the use and scale of distributed and networked systems, ushering in a variety of global-scale, data intensive applications. Furthermore, as computers become ever-faster and communication bandwidth ever-cheaper, computing and communication capabilities are penetrating every facet of our physical world, giving rise to a new class of Cyber Physical Systems (CPS). The operations of these physical and engineered systems are monitored, coordinated, controlled and seamlessly integrated by an intelligent computing core. This intimate coupling between the cyber and physical will transform how we interact with the physical world. The talk will discuss future research trends, grand challenges, opportunities, and initiatives in computing and networking with a focus on future networking technology and Cyber Physical Systems.
\end{abstract}

